\title{
Effects of High $g$ Values on Growth and Chlorophyll Content in Hydrated and Dehydrated Wheat Seeds
}

\author{
Sagar S. Jagtap ${ }^{1 *}$ and P. B. Vidyasagar ${ }^{2}$ \\ ${ }^{1}$ Department of Physics, Haribhai V. Desai College, Pune-411002, MS, India \\ ${ }^{2}$ Biophysics Laboratory, Department of Physics, Savitribai Phule Pune University, Pune-411007, MS, \\ India \\ Email:*ssjagtap83@gmail.com; prof_pbv@yahoo.com
}

\begin{abstract}
Higher $g$ value stimuli (gravitational acceleration more than $1 \mathrm{~g}$ and referred as hyper gravity) caused by centrifugation have been shown to inhibit elongation growth of various plants. In the present study, effects of high $g$ values were studied on wheat seeds with and without water medium at the time of exposure to high g values. Wheat seeds (variety: LOK-1) were washed with $0.5 \%$ fungicide and then 4-5 times with distilled water $(D / W)$. Seeds were then soaked in $D / W$ for $24 \mathrm{hrs}$. Two different experiments were performed. In initial experiment, soaked seeds were taken into the centrifuge tubes filled with $1 \mathrm{ml} \mathrm{D} / \mathrm{W}$. Seeds were exposed to hypergravity ranging from $500 \mathrm{~g}$ to $2500 \mathrm{~g}$ for 10 minutes. In another experiment, soaked seeds were taken into the centrifuge tubes without water or any other medium and then exposed to high g values. After exposure seeds were immediately sowed on 0.8\% agar gel. Results obtained showed that exposure to high g values suppressed growth in wheat seedlings when seeds were exposed to high $g$ values with water as a medium. Chlorophyll content also decreased with increase in g. However, no change in growth and chlorophyll content were observed when seeds were exposed without water medium up to $g$ values as high as 2500. Thus, effects of high $g$ value stimuli depend not only on how much centrifugal force is applied to the seeds but also depends upon how much force is experienced by the inner part of the seeds. Present observation shows that effective centrifugal force experienced by the seeds is different when applied with and without medium.
\end{abstract}

Key words: Hypergravity, centrifugation, chlorophyll content, wheat

\section{Introduction}

A high acceleration ( $\mathrm{g}$ ) value, which is also referred as hypergravity, can be produced on earth by centrifugation. Acceleration due to centrifugation has been used to analyze the mechanisms of gravity perception and gravicurvature $[1,2]$. Changes in shoot growth have also been studied under hypergravity conditions. Hypergravity stimuli (gravitational acceleration more than $1 \mathrm{~g}$ ) caused by centrifugation have been shown to inhibit elongation growth of pea epicotyls [3], radish and cucumber hypocotyls [4], cress hypocotyls [5], azuki bean epicotyls [6]. Stem elongation in Arabidopsis thaliana inflorescence stems was suppressed while dry weight of the inflorescence stems increased in hypergravity [7, 8]. Lignin formation and cell wall polysaccharide content in cress hypocotyls [4] and hemicellulosic polysaccharides in wheat coleoptiles [9] increased in hypergravity. Similar results have been obtained for maize coleoptiles and mesocotyls when 3 days old 20-25 mm long maize seedlings were exposed to hypergravity i.e. $300 \mathrm{~g}$ for $6 \mathrm{hrs}$ at $25^{\circ} \mathrm{C}$ in dark [6] and for azuki bean epicotyls when 5 days old 30-35 $\mathrm{mm}$ long azuki bean seedlings were exposed to $300 \mathrm{~g}$ for $10 \mathrm{hrs}$ at $25^{\circ} \mathrm{C}$ in dark [10]. In these studies, seedlings were exposed to hypergravity and seedlings were either wrapped with tissue paper or mounted on agar gel at the time of exposure to hypergravity. However, only one report showed decrease in growth and chlorophyll content in rice where seeds were exposed to hypergravity and seeds were exposed to hypergravity with water as a medium inside the centrifuge tubes at the time of hypergravity exposure [11].

Effects of high g values on plants depend upon several factors such as relative centrifugal force (RCF), how long the centrifugal force is applied, shape and size of centrifuge tube, orientation of seeds during centrifugation, properties of medium etc. Depending on seeds orientation at the time of exposure the most affected area can be decided. If the most affected area is the embryo of the seed, suppressed growth will be observed. Properties of medium may also change by centrifugation which could results in difference in growth with and without medium. Here we report the results of effects of hypergravity on wheat seeds with and without water medium at the time of exposure to hypergravity. 


\section{Materials and Methods}

Wheat seeds (variety: LOK-1) were obtained from Sheti Udyog Bhandar, Pune. Seeds were washed with $0.5 \%$ fungicide (Uthane M-45) and then 4-5 times with distilled water (D/W). Seeds were then soaked in $\mathrm{D} / \mathrm{W}$ for $24 \mathrm{hrs}$. Two different experiments were performed. In initial experiment, eight soaked seeds were taken into each of the five centrifuge tubes filled with $1 \mathrm{ml} \mathrm{D} / \mathrm{W}$. Seeds were exposed to hypergravity ranging from $500 \mathrm{~g}$ to $2500 \mathrm{~g}$ for only 10 minutes. In another experiment, 8 soaked seeds were taken into the centrifuge tubes without water inside the tubes and then exposed to hypergravity ranging from $500 \mathrm{~g}$ to $2500 \mathrm{~g}$ for only 10 minutes. After hypergravity exposure seeds were immediately sowed on $0.8 \%$ agar gel. Seeds not exposed to hypergravity acted as a control.

The experiments were carried out under ambient conditions of temperature $\left(23 \pm 4{ }^{\circ} \mathrm{C}\right)$ and humidity $(55 \pm 10 \%)$ and a light intensity of $15 \mu \mathrm{molm}^{-2} \mathrm{~s}^{-1}$ for 16 hours day ${ }^{-1}$. Daily growth was observed. Measurements were carried out on the $5^{\text {th }}$ day after sowing. Seed germination percentage of 5 days old seedlings were calculated. Chlorophyll was extracted by using DMF by using the technique given by Porra [12]. Absorption spectra of chlorophyll were taken using a UV-visible spectrometer (Lambda-950, Perkin Elmer, Singapore) and fluorescence spectra at an excitation wavelength of $440 \mathrm{~nm}$ using a Luminescence spectrometer (LS-55, Perkin Elmer, Singapore) respectively. Chlorophyll a, chlorophyll b and total chlorophyll contents were calculated by using Arnon's formula [13]. Experiments were repeated for three times and consistent results were obtained.

\section{Results}

Figure 1a shows growth of unexposed and exposed wheat seeds to high g values with water as a medium inside the centrifuge tubes at the time of exposure. Results obtained showed that the average seed germination percentage for control, $500 \mathrm{~g}, 1000 \mathrm{~g}, 1500 \mathrm{~g}, 2000 \mathrm{~g}$ and $2500 \mathrm{~g}$ were $92 \%, 92 \%, 71 \%$, $50 \%, 21 \%$ and $21 \%$ respectively. No growth was observed in $2000 \mathrm{~g}$ while few seeds were germinated even at $2500 \mathrm{~g}$. This value is fluctuating that is sometimes no growth was observed at $2000 \mathrm{~g}$ and sometimes at $2500 \mathrm{~g}$. Fig. $1 \mathrm{~b}$ shows histogram plot of shoot lengths and root lengths for control and hypergravity treated samples. As seen from the histogram shoot lengths and root lengths were higher in case of control samples as compared with hypergravity exposed samples $(\mathrm{p}>0.1, \mathrm{p}<0.025, \mathrm{p}<0.001, \mathrm{p}$ $<0.001$ and $\mathrm{p}<0.001$ for $500 \mathrm{~g}, 1000 \mathrm{~g}, 1500 \mathrm{~g}, 2000 \mathrm{~g}$ and $2500 \mathrm{~g}$ respectively).

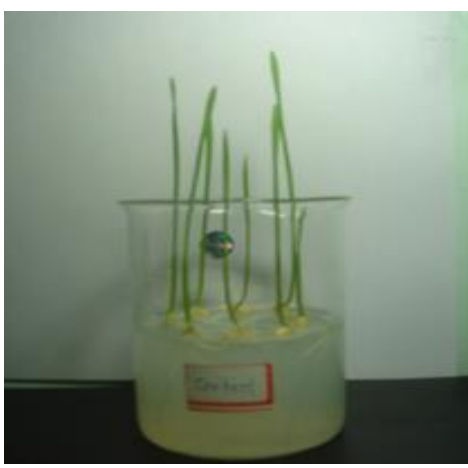

Control

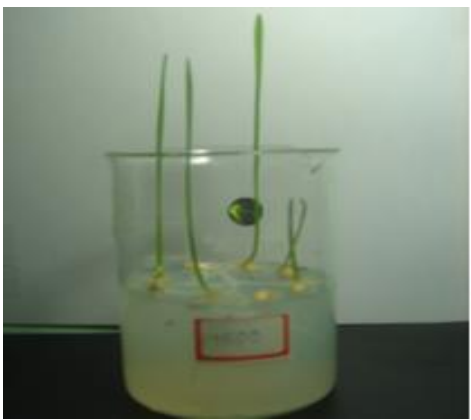

$1500 \mathrm{~g}$

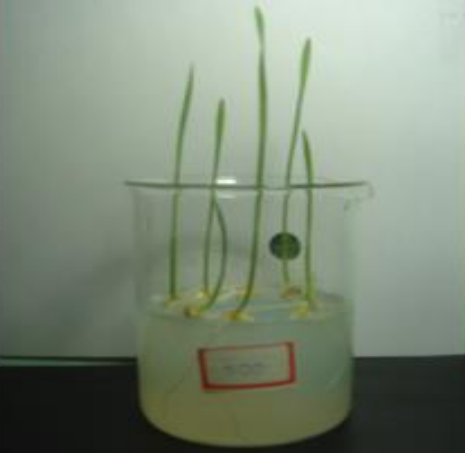

$500 \mathrm{~g}$

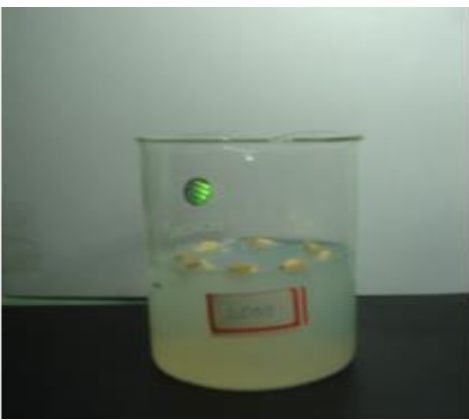

$2000 \mathrm{~g}$

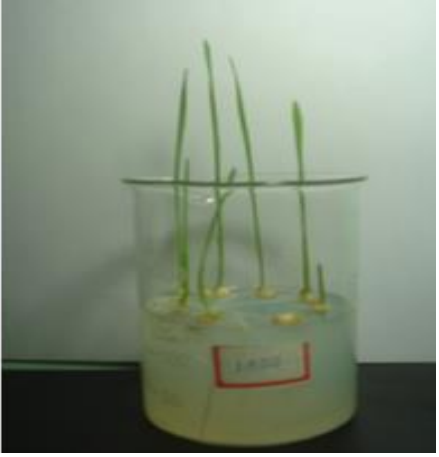

$1000 \mathrm{~g}$

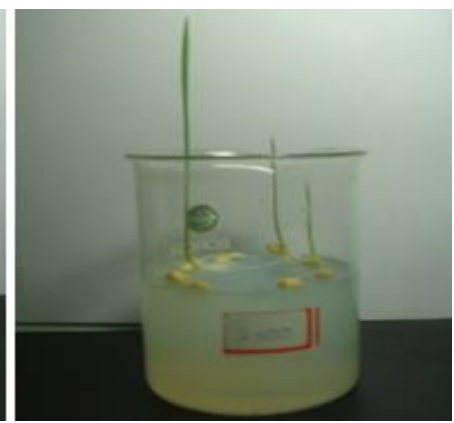

$2500 \mathrm{~g}$

Fig. 1a) 

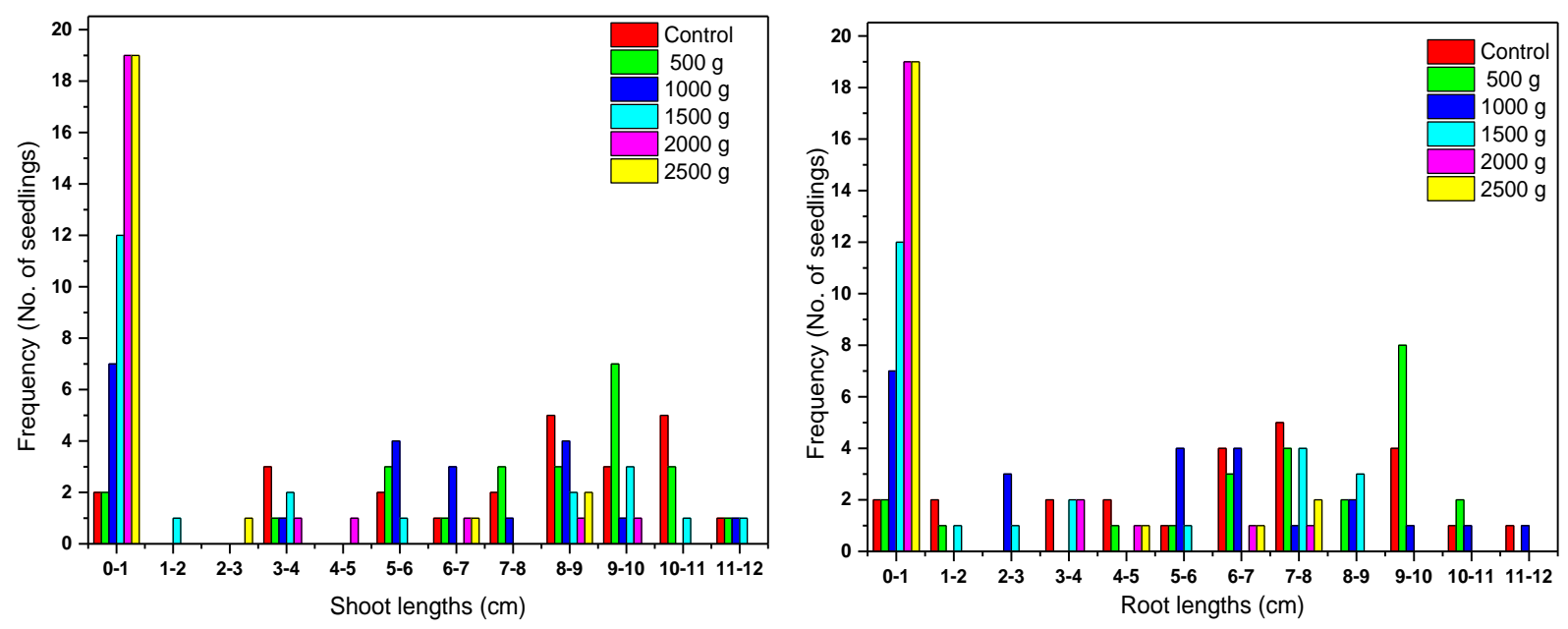

Fig. 1b)

Figure 1. a) Shows growth of unexposed (control) and seeds exposed to higher ' $\mathrm{g}$ ' values (hypergravity) with water medium. These photos are representative of 3 experiments. b) Histogram showing average shoot lengths and average root lengths for control and hypergravity treated samples.

Figure 2a shows absorption spectra of chlorophyll for control and hypergravity exposed samples extracted from shoots of 5 day old seedlings. Absorption spectra of chlorophyll extracted from shoots show two peaks one at $433.5 \mathrm{~nm}$ and another at $669 \mathrm{~nm}$. No shift in wavelength was observed. However, a decrease in the intensity at 433.5 and $669 \mathrm{~nm}$ in case of hypergravity treated samples observed suggested that the chlorophyll content has decreased in hypergravity treated samples except at $500 \mathrm{~g}$ where slight increase in chlorophyll content was observed (Fig. 2b, Table 1).

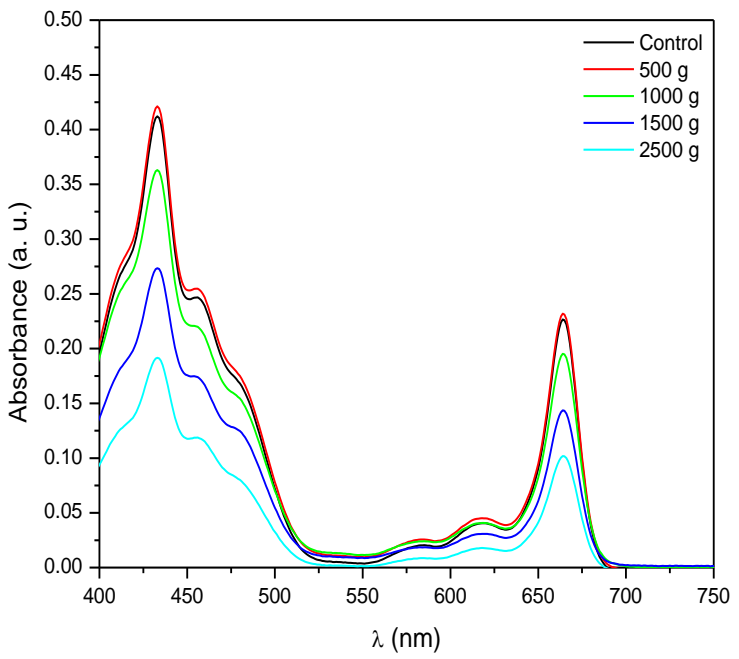

Fig. 2a)

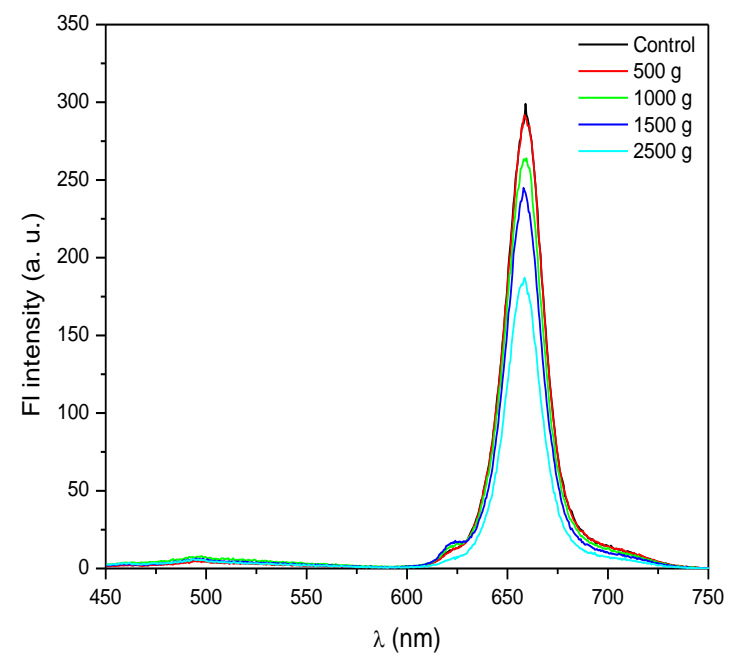

Fig. 2b) 


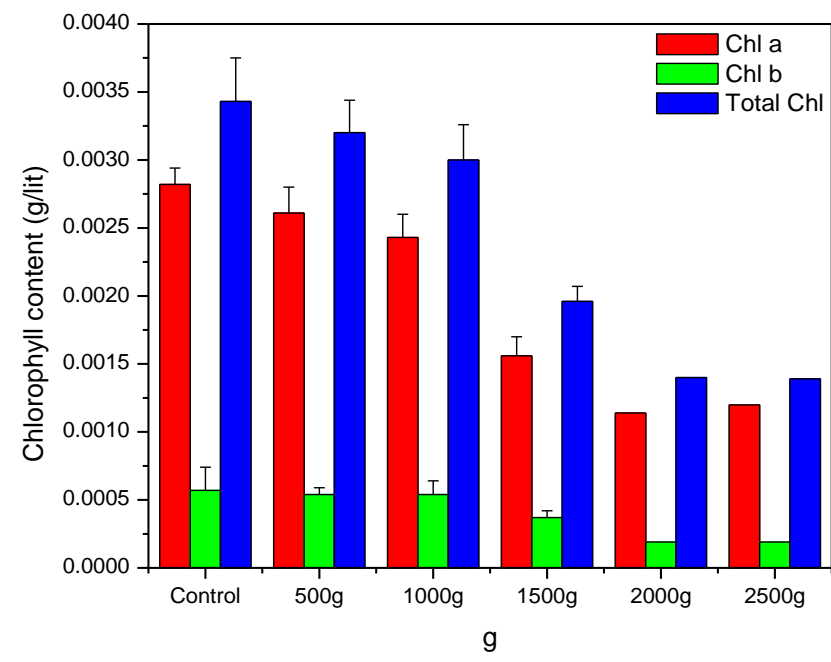

Fig. 2c)

Figure 2. a) Absorption and b) fluorescence spectra of chlorophyll for unexposed (control) and hypergravity treated samples with water. These spectra are a representative of 3 experiments. c) Chlorophyll content for unexposed (control) and hypergravity treated samples. Average percentage decrease in chlorophyll content in $500 \mathrm{~g}, 1000 \mathrm{~g}, 1500 \mathrm{~g}, 2000 \mathrm{~g}$ and $2500 \mathrm{~g}$ was $7 \%, 13 \%, 43 \%, 59 \%$, and $60 \%$ respectively.

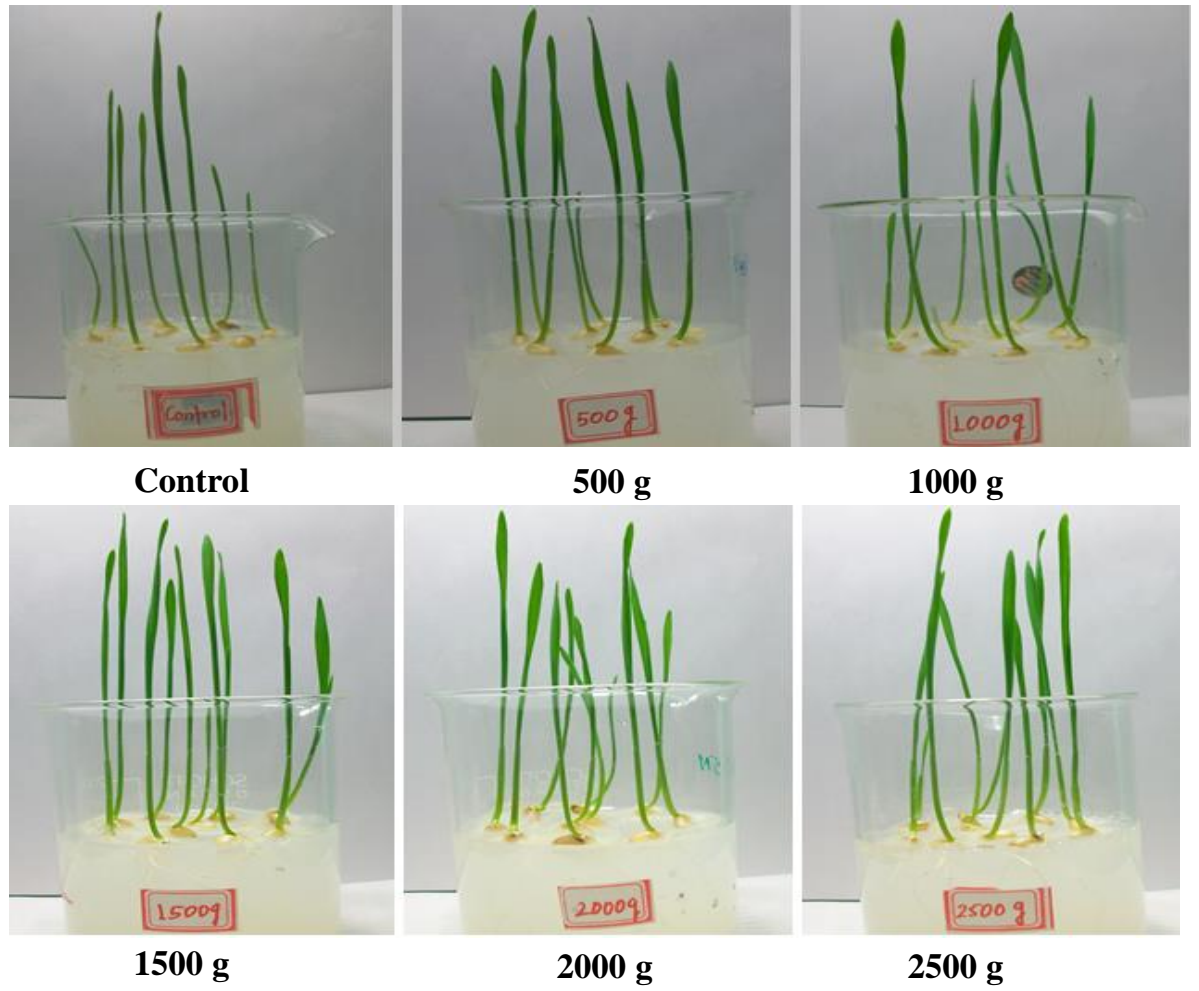

Figure 3. Shows growth of unexposed (control) and seeds exposed to higher ' $g$ ' values (hypergravity) without water inside the centrifuge tubes. These photos are representative of 3 experiments.

Figure 3 shows growth of unexposed and exposed wheat seeds for seeds exposed to hypergravity without water or any other medium inside the centrifuge tubes at the time of exposure. Results obtained showed no change in the average seed germination percentage (100\% in all the samples) for control, 500 $\mathrm{g}, 1000 \mathrm{~g}, 1500 \mathrm{~g}, 2000 \mathrm{~g}$ and $2500 \mathrm{~g}$. Also, no significant change in the root and shoot lengths and weights observed in control and hypergravity treated samples (Table 1). 
Table 1. Average root and shoot lengths and weights for control and hypergravity treated samples. As shown in figure no significant change in root, shoot lengths and weights was observed.

\begin{tabular}{ccccc}
\hline g Value & $\begin{array}{c}\text { Average root } \\
\text { length }(\mathrm{cm})\end{array}$ & $\begin{array}{c}\text { Average shoot } \\
\text { length }(\mathrm{cm})\end{array}$ & $\begin{array}{c}\text { Average Root } \\
\text { weight }(\mathrm{gm})\end{array}$ & $\begin{array}{c}\text { Average Shoot } \\
\text { weight }(\mathrm{gm})\end{array}$ \\
\hline Control & $8.27 \pm 0.15$ & $9.28 \pm 0.33$ & $0.37 \pm 0.03$ & $0.42 \pm 0.04$ \\
$500 \mathrm{~g}$ & $8.24 \pm 0.69$ & $8.87 \pm 0.50$ & $0.35 \pm 0.04$ & $0.41 \pm 0.02$ \\
$1000 \mathrm{~g}$ & $8.41 \pm 0.18$ & $8.95 \pm 0.67$ & $0.33 \pm 0.07$ & $0.40 \pm 0.05$ \\
$1500 \mathrm{~g}$ & $8.23 \pm 0.10$ & $9.19 \pm 0.74$ & $0.35 \pm 0.05$ & $0.43 \pm 0.04$ \\
$2000 \mathrm{~g}$ & $8.44 \pm 0.36$ & $8.67 \pm 0.51$ & $0.35 \pm 0.06$ & $0.40 \pm 0.03$ \\
$2500 \mathrm{~g}$ & $8.40 \pm 0.33$ & $9.13 \pm 0.77$ & $0.35 \pm 0.06$ & $0.42 \pm 0.03$ \\
\hline
\end{tabular}

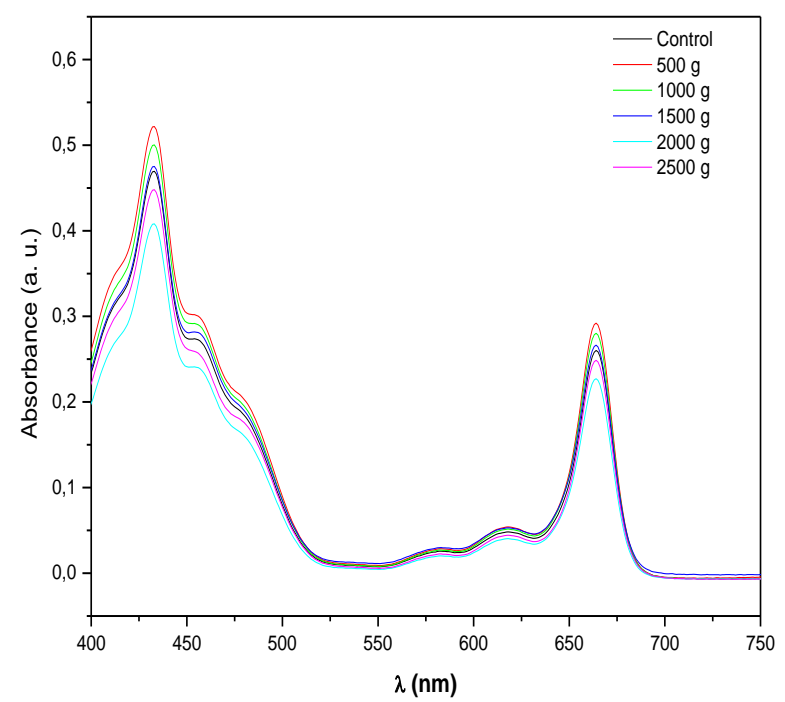

Fig. 4a)

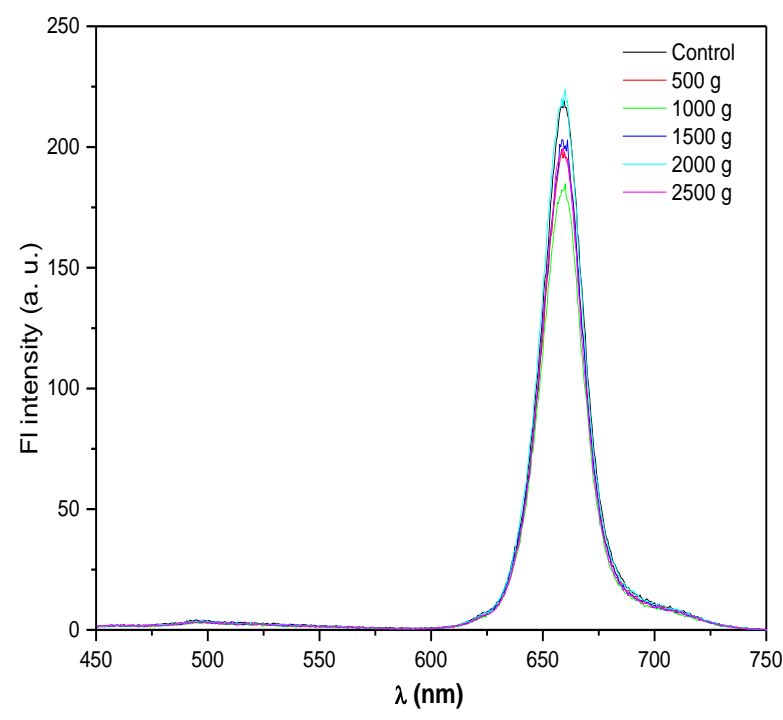

Fig. 4b)

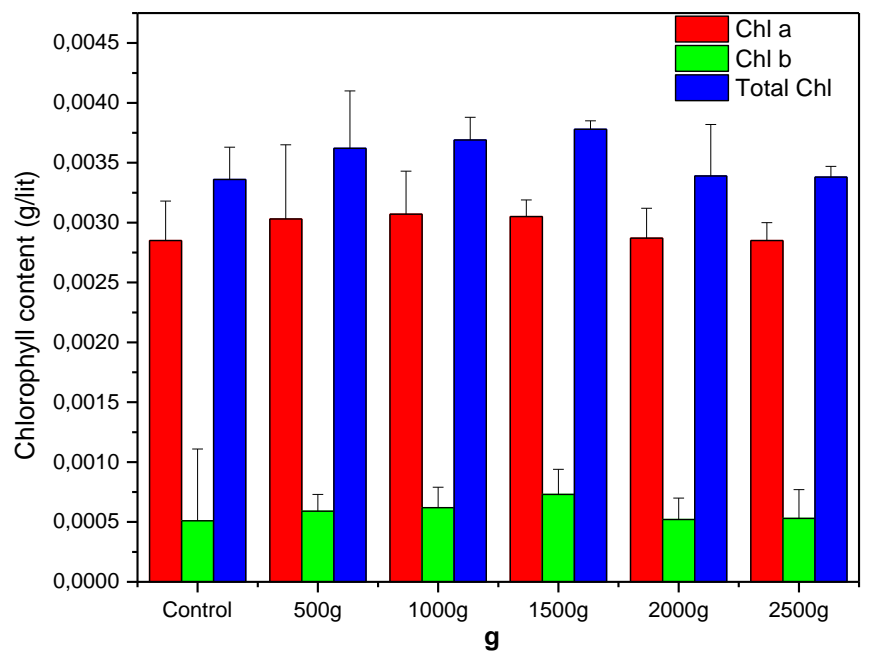

Fig. 4c)

Figure 4. a) Absorption and b) fluorescence spectra of chlorophyll for unexposed (control) and hypergravity treated samples. These spectra are a representative of 3 experiments. c) Chlorophyll content for unexposed (control) and hypergravity treated samples. As shown in figure no significant change in chlorophyll content was observed.

As seen from the Fig. $4 \mathrm{a}$ and $4 \mathrm{~b}$, chlorophyll a, chlorophyll b and total chlorophyll contents were also not changed significantly in hypergravity treated samples as compared to control samples. 


\section{Discussion}

The decrease in seed germination percentage and also decrease in root and shoot lengths for seeds exposed to hypergravity with water as a medium inside the test tubes at the time of exposure suggests that hypergravity suppresses plant growth. This is in agreement to those results obtained for Arabidopsis [7], pea [3], maize [6] and cress [5]. No growth was observed in $2000 \mathrm{~g}$. Some of the seeds germinated even at $2500 \mathrm{~g}$ indicated that seeds orientation was not proper at the time of hypergravity exposure. Decrease in chlorophyll content was observed in hypergravity samples. This is in consistent with the results obtained for rice [11] and wheat $[14,15]$. However, no change in seed germination percentage, growth and chlorophyll content was observed for seeds exposed to hypergravity without water or any medium inside the test tubes at the time of exposure. These results suggest that effects of hypergravity on growth and chlorophyll content in plants depend on medium through which hypergravity stress.

According to the sedimentation theory, particle in a centrifugal field will experience a centrifugal force defined by:

$$
F_{c}=m \omega^{2} r
$$

Where, $F_{c}$ is the centrifugal force, $\mathrm{m}$ is the mass of the particle, $\omega$ is angular velocity and $r$ is the distance from the axis.

This force will be opposed by a buoyant force $\left(F_{b}\right)$ and a frictional force $\left(F_{f}\right)$. The buoyant force represents the force it takes to displace solvent as the particle moves through the centrifugal field. The frictional force represents the drag on the particle as it passes solvent molecules. These two forces are respectively defined as,

$$
F_{b}=-m_{o} \omega^{2} r \text { and } F_{f}=-f v
$$

Where, $m_{o}$ is the mass of the displaced solution, $f$ is a frictional coefficient and $v$ is velocity of the particle.

The particle will move at a velocity such that the total force equals 0 , thus

$$
F_{c}+F_{b}+F_{f}=0 \text { or } m \omega^{2} r-m_{o} \omega^{2} r-f v=0
$$

by replacing $m_{0}$ with $m V \rho s$ in equation (3) where $V$ is partial specific volume of the particle and $\rho s$ is density of the solvent, and solving we get

$$
m \omega^{2} r=\frac{f v}{1-\mathrm{V} \rho \mathrm{s}}
$$

This equation tells us that, the denser the solution (medium), the higher the centrifugal force experienced by the particle. Thus, the centrifugal force experienced by the seeds was different when seeds were exposed with water medium and without water medium inside the tubes. Further calculation gives net centrifugal force experienced by the seeds with water was approximately 4 times greater than centrifugal force experienced by seeds without water.

\section{Conclusion}

Effects of hypergravity stimuli depend not only on how much centrifugal force is applied to seeds but also depends upon how much force is experienced by seeds. Centrifugal force experienced by the seeds is different if it is applied through air and the water. Therefore, the growth and chlorophyll content were retarded in hydrated seeds than dehydrated seeds when exposed to higher g values.

\section{Acknowledgment}

Both the authors thankful to Department of Physics, Savitribai Phule Pune University for providing necessary experimental facilities to carry out this research. First author thanks to PGK Mandal authorities for their constant support and timely help to conduct this research.

\section{References}

[1] Sievers A and Heyder-Caspers L, The effect of centrifugal accelerations on the polarity of statocytes and on the graviperception of cress roots. Planta, vol. 157, 1983, pp. 64-70. 
[2] Fitzelle KJ and Kiss JZ, Restoration of gravitropic sensitivity in starch-deficient mutants of Arabidopsis by hypergravity. J. Exp. Bot., vol. 52, 2001, pp. 265-275.

[3] Waldron KW and Brett CT., Effects of extreme acceleration on the germination, growth and cell wall composition of pea epicotyls. J Exp Bot., vol. 41, 1990, pp. 71-77.

[4] Kasahara HM, Shiwa Y, et al., Effects of hypergravity on elongation growth in radish and cucumber hypocotyls. J. Plant Res., vol. 108, 1995, pp. 59-64.

[5] Hoson T, Nishitani K, et al., Effects of hypergravity on growth and cell wall properties of cress hypocotyls. J Exp Bot., vol. 47 (297), 1996, pp. 513-517.

[6] Soga K, Harada K, et al., Increased molecular mass of hemicellulosis polysaccharides is involved in growth inhibition of maize coleoptiles and mesocotyls under hypergravity conditions. J Plant Res., vol. 112, 1999a, pp. 273-278.

[7] Tamaoki D, Karahara I, et al., Effects of hypergravity conditions on elongation growth and lignin formation in the inflorescence stem of Arabidopsis thaliana. J Plant Res., vol. 119, 2006, pp. 7984.

[8] Nakabayashi I, Karahara I, et al., Hypergravity stimulus enhances primary xylem development and decreases mechanical properties of secondary cell walls in inflorescence stems of Arabidopsis thaliana. Annals Bot., vol. 97, 2006, pp. 1083-1090.

[9] Wakabayashi, K, Soga, K, et al., Increase in the level of arabinoxylan-hydroxycinnamate network in cell walls of wheat coleoptiles grown under continuous hypergravity conditions. Physiol. Plant., vol. 125 (1), 2005, pp. 127-134.

[10] Soga, K., Wakabayashi, K., et al. Hypergravity increases the molecular mass of xyloglucans by decreasing xyloglucan-degrading activity in azuki bean epicotyls. Plant Cell Physiol., vol. 40 (6), 1999b, pp. 581-585.

[11] Vidyasagar P, Jagtap S, et al., Effects of hypergravity on the chlorophyll content and growth of root and shoot during development in rice plants. Photosynthesis Energy from the Sun. Springer Netherlands, 978-1-4020-6707-5 (Print) 978-1-4020-6709-9 (Online), vol. 2, 2008, pp. 1599-1602.

[12] Porra, R. J., Thompson, W. A., et al., Determination of accurate extinction coefficients and simultaneous equations for assaying chlorophylls $a$ and $b$ extracted with four different solvents: verification of the concentration of chlorophyll standards by atomic absorption spectroscopy. Biochem. Biophys. Acta., vol. 975, 1989, pp. 384-394.

[13] Arnon DI, Copper enzymes in isolated chloroplasts. Polyphenoloxidase in Beta vulgaris. Plant Physiol., vol. 24 (1), 1949, pp. 1-15.

[14] Sagar S. Jagtap and Pandit B. Vidyasagar "Effects of high gravity (g) values on growth and chlorophyll content in wheat", Int. J. Integ. Biol., vol. 9 (3), 2010, pp.127-129.

[15] Pandit Vidyasagar, Sagar S. Jagtap, Jyotsana P. Dixit, Shailendra M. Kamble, Aarti P. Dhepe, Effects of Short Interval High 'g' Exposure on Germination, Growth and Photosynthesis of Triticum aestivum L. Microg Sci. \& Tech., vol. 26 (6), 2014, pp. 375-384. 